\title{
Prosthetic Cardiac Valve Type
}

National Cancer Institute

\section{Source}

National Cancer Institute. Prosthetic Cardiac Valve Type. NCI Thesaurus. Code C139051.

A description of the type of artificial cardiac valve in use. 